\title{
Microsurgery: A Useful and Versatile Tool in Surgical Field
}

\author{
Mahendra Singh* and Ashish Saxena \\ Department of Surgery, Hindu Rao Hospital, Delhi, India
}

\begin{abstract}
Microsurgery includes various surgeries which are performed with the assistance of microscope and special miniaturized instruments. Almost all the subspecialties of surgery have benefitted by utilizing the techniques of microsurgery. It is used in tissue reconstruction after trauma or tumor resection to achieve a functionally and cosmetically superior outcome. It has also found application in precise resection of malignancies such as skin tumors and laryngeal cancers. Recent advances in technology have made microsurgery an efficient tool for surgeons to deal with various diseases. Despite having a steep learning curve, the advantages of microsurgery over the conventional surgical methods are unquestionable and include the cleaner and smaller incisions, lesser hemorrhage, minimal tissue handling and a closer wound approximation.
\end{abstract}

Keywords: Microsurgery; Skin tumors; Laryngeal cancers; Lesser hemorrhage

\section{Introduction}

Microsurgery is an evolving discipline of surgery that deals with the surgery of very small structures such as blood vessels and nerves typically $1 \mathrm{~mm}$ or less in diameter [1]. Despite its relatively recent advent it has created its own niche in the various domains of surgery in no time. Be it the field of vascular surgery, neurosurgery, pediatric surgery, orthopaedic surgery, ophthalmic surgery or plastic and reconstructive surgery, everywhere microsurgery is gaining popularity [1]. Hence it becomes essential for surgeons to have a basic conceptual knowledge of this discipline.

Broadly speaking, any surgery which is performed with assistance of an operating microscope or surgical loupes comes under the realm of microsurgery [1]. It often requires use of miniaturized instruments which are manipulated in magnified visual field obtained by the microscopes. Modern day procedures that utilize microsurgical techniques include nerve repair, nerve grafting, blood vessel repair, vascular grafting, free tissue transfers, and replantation of severed limbs and digits.

\section{History of Microsurgery}

The evolution of microsurgery started in the beginning of $20^{\text {th }}$ century. Carl Nylen, who is known as the father of microsurgery, was first to use a binocular microscope to correct otosclerotic deafness in 1921 [2]. In 1960, Jules Jacobson performed first microvascular surgery using a microscope for repairing blood vessels. He also coined the term 'Microsurgery' [3]. Malt and McKhann pioneered the reconstruction surgery by replantation of amputated arms in 1962 [4]. Harold Kleinert and Mort Kasdan reported first successful revascularization of a partially amputated digit in 1963 [5]. In 1964, Nakayama reported the first case series of microsurgical free tissue transfer of vascularized intestinal segments for the oesophageal reconstruction [6]. Contemporary reconstructive microsurgery was introduced by Buncke and Schulz in 1964. They successfully performed experimental replantation of rabbit ears and monkey digits [7]. In 1968, Cobbett transferred a great toe to replace an amputated thumb [8]. In recent years focus has been on the assimilation of modern technology such as LASER, electric energy into routine microsurgery.

\section{Basic Principles of Microsurgery}

Principles of microsurgery are based upon three essential components forming the Triad of microsurgery. These include magnification, illumination and instruments [9]. Proper coordination between these components is vital for the optimal outcome and they are discussed below.

\section{Magnification}

Greater the size of image, greater is the ability of eye to appreciate the minute details of the object. Two obvious ways to increase the image size are either by getting closer to the object or by magnification. The extent to which eyes can get closer to object gets significantly limited by the power of accommodation. Therefore the only practical method for increasing the image size is magnification. Magnification in clinical practice is obtained either by loupes or surgical microscopes. Initially magnifying lenses (loupes) were used which were later replaced by surgical microscopes. In loupes, magnification and focal length (working distance) are fixed while both are adjustable in surgical microscopes [10]. These microscopes have foot controls to adjust focus and magnification so that surgeon's hands remain free to manipulate the instruments. They may be of single, dual or multiple viewer type. A magnification of $5 \mathrm{x}$ to $40 \mathrm{x}$ is required in most of the microsurgical procedures. For dissection $4.7 x$ to $7.8 x$ magnification is commonly used, while for the purpose of anastomosis a magnification range of $7.8 \mathrm{x}$ to $12.5 \mathrm{x}$ is used [10].

\section{Illumination}

Collateral lighting systems with suitable fixing apparatus are helpful for higher magnification. The important qualities to be considered while choosing an appropriate illumination source are its weight, brightness of light, and the ease of focusing and directing the light [11].

\section{Instruments}

Microsurgical instruments need to have an ergonomic design for precise handling for long periods. They are therefore made circular in cross section to allow the rotational movements by the operator. The instruments have pencil-hold type of grip not the conventional palm-hold grip as seen in general surgical instruments. Being made of titanium they are intrinsically strong, light weight and non magnetic. They should be approximately $18 \mathrm{~cm}$ long and lie on the saddle between the operator's thumb and index finger. Their top ends are slightly heavy to facilitate accurate handling. They should ideally be color

*Corresponding author: Mahendra Singh, Department of Surgery, Hindu Rao Hospital, Delhi, India, Tel: +91 112391 9476; E-mail: dr.mahi1118@gmail.com

Received March 19, 2014; Accepted April 16, 2014; Published April 18, 2014

Citation: Singh M, Saxena A (2014) Microsurgery: A Useful and Versatile Tool in Surgical Field. Surgery Curr Res 4: 194. doi:10.4172/2161-1076.1000194

Copyright: ( 2014 Singh M, et al. This is an open-access article distributed under the terms of the Creative Commons Attribution License, which permits unrestricted use, distribution, and reproduction in any medium, provided the original author and source are credited. 
coated to avoid the metallic glare. Needle holder should be equipped with a precise working lock that should not exceed a locking force of $50 \mathrm{~g}$ as high locking forces generate tremor and low locking forces reduce feeling for movement. The instruments include forceps, needle holders, scissors, vascular clamps, irrigators and vessel dilators [12]. It is advisable to have the hands and forearms supported by the table or other fixed items. Specially designed suture material of gauges 9-0 to $12-0$ and needles with a diameter of $<0.15 \mathrm{~mm}$ are used [13]. The color of suture material is very important since non colored material is invisible under magnification. Hence very dark-tinted sutures are used.

\section{Fundamental Techniques in Microsurgery}

\section{Free tissue transfer/flap}

Free tissue transfer is a surgical reconstructive procedure using microsurgery. In this a region of donor tissue is selected which can be isolated upon a feeding artery and vein. Free tissue flaps can be categorized into isolated and composite types. Isolated flaps consist of skin, fascia, nerve, muscle or bone taken individually, whereas composite flap is a combination of more than one tissue type. They include myo-cutaneous, osteo-cutaneous, or innervated myocutaneous flaps $[14,15]$.

The flap thus harvested from donor site is now transferred to recipient site requiring reconstruction and vessels of the free flap are anastomosed to vessels of the recipient site by microsurgical methods.

\section{Replantation}

The reattachment of a completely detached body part is known as replantation. Most commonly replanted organs are fingers and thumbs but the ear, scalp, nose, face, arm and penis replantation have also been reported. Replantation involves restoration of the blood flow, musculoskeletal system and the nerves as required [15]. Functional demands of amputated specimen are paramount in replantation. According to modern concept the survival alone of replanted part is not sufficient. The functional utility of organ also has to be taken into account and has to be weighed against the patient's ability to tolerate long rehabilitation process, both on physical and psychological levels.

Similarly it is now understood that an amputated organ can be transferred to a new site in order to enhance functional outcome of surgery. For example, a finger or a great toe can be transposed in position of a non-salvageable amputated thumb to obtain a more functional hand [8]. Such types of procedures are known as "Spare part surgery" [3].

\section{Transplantation}

Microsurgery represents the link between surgery and transplant immunological research. First microsurgical experiments in transplantaion (porto-caval anastomosis in the rat) were performed by Dr. Sun Lee a pioneer in the field of microsurgery [16]. Microsurgical techniques have played a crucial role in the field of transplantation research because it allowed use of rodent models. It was also important to develop new techniques of transplantation that would be later performed in humans. In addition, it allows reconstruction of small arteries in clinical organ transplantation.

\section{Various Procedures in Microsurgery}

Microsurgical reconstructive technique can be either orthoplastic or oncoplastic. In orthoplastic procedures, various free flaps are used to reconstruct or replace the damaged structures such as face, digit and limbs. The oncoplastic technique is utilized in the case of malignancy where reconstruction is required after wide excision of growth. For example, Deep Inferior Epigastric Perforator (DIEP) flap is used in breast malignancies. Otolaryngologists perform microsurgery on structures of inner ear or vocal cords [2]. Ophthalmologists perform cataract surgery, corneal transplants, and treatment of conditions like glaucoma with assistance of microsurgery. Urologists and gynecologists reverse vasectomies and tubal ligations to restore fertility using microsurgical techniques. Orthopaedic surgeons perform lumbar microsurgery for delicate procedures like removal of diseased portion of bone or intervertebral discs [17]. Microsurgery can play a life saving role in the field of neurosurgery where tumors can be removed and vascular anomalies in brain can be corrected.

\section{Recent Advances in Microsurgery}

\section{TOMS-Three Dimensional On-Screen Microsurgery System}

It facilitates three dimensional visualization of (micro) surgical field on a video monitor, without need to visualize physically through the microscope [18]. It provides a clear stereoscopic view with accurate sense of depth perception.

\section{HDTV single camera 3D system}

This involves a high definition television attached to the microscope which enables three dimensional visualization and documentation as well [19].

\section{Supermicrosurgery}

The highly challenging task of anastomosis of very small vessels $(<0.3 \mathrm{~mm})$ is now possible with the assistance of modern optical equipments [20]. This is known as supermicrosurgery. It has been used in the anastomosis of perforator flaps.

\section{Trans oral Laser Microsurgery (TLM)}

It is a recent type of minimally invasive surgery for oral cancers, especially in laryngeal cancer. It employs $\mathrm{CO}_{2}$ laser via endoscopic approach. TLM follows an incisional resection technique under microscopic guidance. The dissection proceeds gradually bit by bit allowing the operator to precisely follow the anatomical interface between tumor and normal tissue. It thus facilitates a greater preservation of normal tissue structure and function [21].

\section{Transanal Endoscopic Microsurgery (TEMS)}

TEMS is a useful micro invasive technique to resect certain large or sessile adenomas of rectum. It is essentially a form of laparoscopic surgery performed in a much more confined space [22].

\section{Electromicrosurgery}

This modality is used in cosmetic procedures like the destruction of feeding vessels in a skin tag, warts, and spider naevi. In it under the guidance of a magnifying lamp, the tip of the finest smallest probe possible, about the size of an eyelash is introduced along the line of a capillary and a tiny energy current is discharged [23].

\section{Mohs micrographic surgery}

This is a highly specialized surgical technique to treat certain types of skin cancers. In it, the skin cancer is removed a thin layer at a time with a very small margin of healthy skin around it. The resected specimen is immediately evaluated under microscope to find out positive margins. The corresponding area of apparently healthy skin is then resected. The entire process is repeated until no residual tumor is left behind [24]. 


\section{Limitations of Microsurgery}

Microsurgery is technically a demanding procedure and has a steep learning curve. Establishment of the set-up is costly. Other difficulties include a restricted area of vision, loss of depth perception and loss of visual reference points [25].

\section{Conclusion}

Microsurgery has become an indispensible tool in virtually all fields of surgery and holds a promising future. It offers several advantages over the conventional surgery such as cleaner and smaller incisions, lesser hemorrhage, minimal tissue handling and a closer wound approximation. These factors also render the post operative period less painful and in turn lead to a faster recovery and a more satisfying outcome. Although one has to be extremely vigilant in patient selection, pre-operative planning, per-operative finesse and post operative monitoring, the rewards gained in the form of excellent results by far outweigh the efforts employed.

\section{References}

1. Daniel RK (1979) Microsurgery: through the looking glass. N Engl J Med 300 1251-1257.

2. Mudry A (2000) The history of the microscope for use in ear surgery. Am J Otol 21: 877-886.

3. Tyagi S, Kumar S (2010) Microsurgery: An important tool for reconstructive surgery- a clinical review. Int J Pharm Bio Sci.

4. Malt Ra, Mckhann C (1964) Replantation of Severed Arms. JAMA 189: 716722

5. kleinert He, Kasdan M (1963) Restoration of Blood Flow in Upper Extremity Injuries. J Trauma 3: 461-476.

6. Nakayama K, Yamamoto K, Tamiya T, Makino H, Odaka M, et al. (1964) Experience with Free Autografts of the Bowel with a New Venous Anastomosis Apparatus. Surgery 55: 796-802.

7. Buncke HJ Jr, Schulz WP (1966) Total ear reimplantation in the rabbit utilising microminiature vascular anastomoses. Br J Plast Surg 19: 15-22.

8. Cobbett JR (1969) Free digital transfer. Report of a case of transfer of a great toe to replace an amputated thumb. J Bone Joint Surg Br 51: 677-679.

9. Kim S, Pecora G, Rubinstein RA (2001) Comparison of traditional and microsurgery in endodontics. In: Color Atlas of Microsurgery in Endodontics. WB Saunders Company, Philadelphia, USA.

10. Schoeffl H, Lazzeri D, Schnelzer R, Froschauer SM, Huemer GM (2013) Optical magnification should be mandatory for microsurgery: scientific basis and clinical data contributing to quality assurance. Arch Plast Surg 40: 104-108.

11. Gu M, Bao H, Kang H (2014) Fibre-optical microendoscopy. J Microsc 254: 13-18.

12. Chang WC, Sretavan DW (2007) Microtechnology in medicine: the emergence of surgical microdevices. Clin Neurosurg 54: 137-147.

13. Furka I, Brath E, Nemeth N, Miko I (2006) Learning microsurgical suturing and knotting techniques: comparative data. Microsurgery 26: 4-7.

14. Shenaq SM, Klebuc MJ, Vargo D (1995) Free-tissue transfer with the aid of loupe magnification: experience with 251 procedures. Plast Reconstr Surg 95 261-269.

15. Morrison WA, McCombe D (2007) Digital replantation. Hand Clin 23: 1-12

16. Lee SH, Fisher B (1961) Portacaval shunt in the rat. Surgery 50: 668-672.

17. Wang M, Zhou Y, Wang J, Zhang Z, Li C (2012) A 10-year follow-up study on long-term clinical outcomes of lumbar microendoscopic discectomy. J Neurol Surg A Cent Eur Neurosurg 73: 195-198.

18. Franken RJ, Gupta SC, Banis JC Jr, Thomas SV, Derr JW, et al. (1995) Microsurgery without a microscope: laboratory evaluation of a threedimensional on-screen microsurgery system. Microsurgery 16: 746-751.

19. Ryo M, Schigeaki K (2001) HDTV single camera 3D system and its application in microsurgery. Sterioscopic display and virtual reality systems. Proc SPIE 2177: 31-34.

20. Koshima I, Nanba Y, Tsutsui T, Takahashi Y (2003) Medial plantar perforator flaps with supermicrosurgery. Clin Plast Surg 30: 447-455, vii.

21. Pearson BW, Salassa JR (2003) Transoral laser microresection for cancer of the larynx involving the anterior commissure. Laryngoscope 113: 1104-1112.

22. Platell C, Denholm E, Makin G (2004) Efficacy of transanal endoscopic microsurgery in the management of rectal polyps. J Gastroenterol Hepatol 19: 767-772.

23. Groot DW, Johnson PA (1987) Lasers and advanced dermatological instrumentation. Australas J Dermatol 28: 77-85.

24. Schell AE, Russell MA, Park SS (2013) Suggested excisional margins for cutaneous malignant lesions based on Mohs micrographic surgery. JAMA Facial Plast Surg 15: 337-343.

25. Ghousia A, Prabhuji MLV, Lavanya R (2012) Microsurgery: A Clinical Philosophy for Surgical Craftsmanship. Ejournal of Dentistry 2: 233-237. 